\title{
PROCESO DE OCUPACIÓN INFORMAL DEL TERRITORIO Y SU INFLUENCIA EN EL PAISAJE HISTÓRICO - CULTURAL CASO: CERRO INTIORKO, DISTRITO ALTO DE LA ALIANZA, CIUDAD DE TACNA 2003 AL 2017.
}

\author{
PROCESS OF INFORMAL OCCUPATION OF THE TERRITORY AND ITS \\ INFLUENCE IN THE HISTORICAL LANDSCAPE - CULTURAL CASE: CERRO \\ INTIORKO, DISTRITO ALTO DE LA ALIANZA, CITY OF TACNA 2003 TO 2017.
}

KEILY NORKA MEDINA BEJAR 1

\section{Resumen}

La ciudad de Tacna presenta un paisaje histórico cultural, que deviene de la guerra con Chile de 1879, dejando como huella a la Cripta de los Héroes, en lo alto del Cerro Intiorko, frente a la ciudad, en busca de rendir homenaje a los caídos en el Campo de la Alianza, a partir de 1901, con la realización de diferentes ceremonias protocolares que contaban con la participación de toda la población tacneña ferviente patriota, hasta el año de 1979. La presente investigación tiene como objetivo determinar la influencia del proceso de ocupación informal del territorio en el paisaje histórico cultural del cerro Intiorko, en el distrito de Alto de la Alianza, de la ciudad de Tacna. La investigación centró su metodología, bajo un enfoque cuantitativo, tipo básico, de diseño no experimental, con una hipótesis descriptiva - explicativa, aplicando la estadística. Se aplicó el cuestionario como instrumento de medición; que permitió recoger información, relacionar y medir las variables de estudio y sus dimensiones. La muestra de estudio estuvo conformada por las 359 parcelas de las asociaciones pecuarias del área de estudio; las variables coadyuvan a la evaluación del paisaje, en base a su análisis desde el enfoque visual, basado en los lineamientos establecidos por la normatividad española. Los resultados obtenidos permitieron establecer que: la ocupación informal del territorio influye en la alteración del paisaje, en la disminución de su calidad visual, con base en la evaluación del paisaje visualizado; ello debido a la disminución del área natural con respecto del área ocupada por la actividad antrópica, con el riesgo de ocupar el espacio correspondiente a la

\section{Abstract}

The city of Tacna presents a historical cultural landscape, which comes from the war with Chile of 1879 , leaving as a footprint the Crypt of the Heroes, high in the Intiorko Hill, in front of the city, in search of paying homage to the fallen In the Field of the Alliance, from 1901, with the accomplishment of different protocol ceremonies that counted on the participation of all the population tacneña fervent patriot, until the year of 1979. The present investigation aims to determine the influence of the process of Informal occupation of the territory in the historical cultural landscape of the hill Intiorko, in the district of Alto de la Alianza, in the city of Tacna. The research focused its methodology, under a quantitative approach, basic type, non - experimental design, with a descriptive - explanatory hypothesis, applying the statistics. The questionnaire was applied as a measurement instrument; Which allowed to gather information, relate and measure study variables and their dimensions. The study sample consisted of the 359 plots of the livestock associations of the study area; The variables contribute to the evaluation of the landscape, based on its analysis from the visual approach, based on the guidelines established by Spanish regulations. The obtained results allowed to establish that: the informal occupation of the territory influences in the alteration of the landscape, in the diminution of its visual quality, based on the evaluation of the visualized landscape; Due to the reduction of the natural area with respect to the area occupied by the anthropic activity, with the risk of occupying the space corresponding to the Crypt of the Heroes,

1 MAESTRO EN ARQUITECTURA PAISAJISTA 
Cripta de los Héroes, es por ello que una planificación territorial que permita el crecimiento de la ciudad de manera ordenada, sostenible a mediano y largo plazo es de suma importancia para el desarrollo de una ciudad.

Palabras clave: Proceso de ocupación, calidad visual, planificación territorial. that is why a territorial planning that allows the growth of the city in an ordered way, Sustainable in the medium and long term is of utmost importance for the development of a city.

Keywords: Occupation process, visual quality, territorial planning.

\section{INTRODUCCIÓN}

La presente investigación aborda el tema del paisaje desde su enfoque visual, considerando el recurso paisajístico que alberga la Cripta de los Héroes, lugar de conmemoración a la guerra del Pacífico de 1879. El área de estudio se encuentra ubicado en el cerro Intiorko, frente a la ciudad, en el distrito de Alto de la Alianza, de la provincia y departamento de Tacna. El crecimiento urbano propio del siglo XXI, produce la ruptura de la relación de los hombres con su entorno, de la identidad-nexo. Aponte (2003).

El cerro Intiorko, es susceptible del fenómeno demográfico, como es la migración, al ocuparse su territorio de manera informal y con el pasar de los años, este ha sido formalizado por el Ministerio de Vivienda a través de programas de inversiones a partir del año 2010. Según el actual instrumento de planificación, Plan de Desarrollo Urbano 2015 - 2025, supervisado por el Ministerio de Vivienda; el área de estudio presenta una zonificación con características especiales, Z-10 pecuario, encontrándose destinado para actividades pecuarias, las mismas que cuentan con áreas de aporte urbano como educación, recreación y otros, según el plano catastral de la Municipalidad Distrital de Alto de la Alianza. No es compatible para dichos usos; difiriendo de lo precisado por el Plan Director de Tacna 2001 y 2010 y el equipo técnico de el Plan de Acondicionamiento Territorial-Plan de Desarrollo Urbano 2014-2023.

La Cripta de los héroes, es una manifestación material que forma parte del paisaje histórico cultural del territorio, dicho inmueble, es evidencia de ser el lugar donde; desde 1901 hasta 1979; se rindió homenaje en gratitud y conmemoración a los caídos en la Batalla del Alto de la Alianza, llevada a cabo el 26 de mayo de 1880. Las ceremonias eran organizadas por el Consejo Provincial de Tacna, con la colaboración del glorioso regimiento Húsares de Junín, con asistencia de autoridades civiles y militares, instituciones sociales y patrióticas, policía, colegios nacionales, escuela normal y escuelas fiscales. Morales (1991)

Entendiendo así que la historia tiene así una eterna seducción que le es propia. Por ella ningún siglo nos está vedado, la pujanza de nuestra mente puede romper los límites de la debilidad del individuo solo. Pero ese saber que es revivir, evocar, comprender, juzgar o interpretar, no es fácil y no puede ser obtenido sino después de duro esfuerzo, severa técnica, metódica labor (Basadre, 196, p. XLI).

Sánchez (2015). Migraciones internas en el Perú refiere que, en las últimas décadas, el Perú ha experimentado cambios significativos en su realidad social, económica y demográfica; donde las migraciones internas han jugado un rol importante para la configuración de la sociedad actual. Ubicando a Tacna junto Arequipa y Moquegua en el tercer eje, precisando la fuerte presencia migratoria. Estas tres regiones en los últimos doce años vienen creciendo a tasas muy superiores a su incremento demográfico, elevando el PBI per cápita, Tacna en el periodo 2001 - 2012, creció a una tasa promedio anual de $4.2 \%$. Moschella (2012) precisa que los humedales de Ventanilla y de Puerto Viejo han experimentado impactos por los procesos de urbanización en forma negativa. En Ventanilla, la urbanización se caracteriza por iniciar como invasiones informales de pobladores de bajos recursos sobre suelos marginales para la habilitación urbana. Martínez (2017) en Tacna demuestra cómo ha influido la presencia de la explotación superficial de canteras en la degradación del paisaje natural del cerro Arunta. Sus principales resultados arrojan que la modificación de la fisiografía por el movimiento de tierras de la explotación superficial de los recursos. García (2008) en México, precisa que el crecimiento de las ciudades provoca grandes efectos en sus regiones circundantes. Concluye que la expansión Urbana en Ciudad Juárez, ha obedecido a distintos factores principalmente de tipo económico. El paisaje y la configuración urbana actual, muestra mellas cuyo origen ha 
sido la mala praxis de la planeación urbana. Masmela (2010) en Colombia desarrolla un ejercicio metodológico de un análisis del paisaje desde su enfoque visual, basado en los lineamientos establecidos por la normatividad española obteniendo como resultado la importancia del análisis del paisaje como elemento a tener en cuenta en la ordenación territorial.

Según el convenio europeo del paisaje, por "paisaje" se entenderá cualquier parte del territorio tal como la percibe la población, cuyo carácter sea el resultado de la acción y la interacción de factores naturales y/o humanos. El paisaje es una extensión de terreno que se ve desde un sitio o una extensión de terreno considerada en su aspecto artístico. (Diccionario de la real lengua española, 1992). Al situarse al hombre ante su entorno, a través de sus recursos intelectuales y perceptivos, se produce en él una respuesta que se llama paisaje. Según esto, paisaje es el resultado global donde el hombre procesa los estímulos de su entorno. (Arias, 2003:89).

El 26 de mayo del 2011, mediante Decreto Supremo N 002-2011-MC, se aprobó el reglamento para la declaratoria y gestión de los paisajes como Patrimonio Cultural de la Nación. Los estudios de paisaje desde su enfoque visual permiten hacer del hecho perceptivo un dato preciso o un término cualitativo que conduzca a la descripción, clasificación y valoración de un paisaje. Bajo esta metodología de estudio, según Villarino (1985:514) se plantea una triple problemática, la calidad intrínseca del paisaje, la respuesta estética y la adjudicación de valor.

Según la metodología desarrollada por West Riding (1969, citado por Villarino) se definen cinco categorías de paisaje: calidad soberbia, calidad grande, áreas atractivas, áreas monótonas y áreas arruinadas. Las principales dificultades según Dunn (1974; citado por Villarino 1985: 516) radican en la polémica evaluadores versus gente normal como evaluadores de la calidad, pues el observador del paisaje se encuentra con una disposición de ánimo diferente a la del espectador de una fotografía y porque el empleo de fotografías reduce un paisaje tridimensional a un conjunto de formas bidimensionales. Dunn (1974; citado por Villarino 1985: 520-521) recomienda incluir todos los factores relevantes en la determinación del carácter y calidad del paisaje, estos a su vez, deben excluirse mutuamente para eliminar la posibilidad de medir dos veces el efecto de un factor determinado.

Pérez, L; Parra, C; (2004) propone a) Poner de relieve el rol que el patrimonio y el paisaje cultural están jugando en la estimulación del desarrollo territorial y b) Estudiar la temática de la revalorización del patrimonio y del paisaje cultural tanto en su vertiente conceptual (conceptos, criterios guía) o de teorías subyacentes, como también desde la formación y estructuración de estos proyectos de parques patrimoniales (instrumentos, herramientas de intervención).

García E. (2008) afirma que el proceso de ocupación del suelo que ha tenido un asentamiento humano, se evidencia mediante el cambio del paisaje con relación al tiempo; es así que, trazar este cambio, permite cuantificar el grado de perturbación de los hábitats naturales por acción humana. Paradójicamente, el argumento original del proceso de dispersión y difusión urbana tiene su origen en la búsqueda de mejores condiciones ambientales, calidad de vida y precios del suelo más reducidos. Entonces, el modelo de crecimiento discontinuo y disperso es básicamente un modelo espontáneo que se apoya en gran medida de la infraestructura existente, en el que la gestión inmobiliaria y la actuación personal de los distintos agentes resultan decisivas (Cerda, 2007). Es así justamente, que este rápido y generalizado proceso de expansión urbana genera cambios en los usos y coberturas de los suelos naturales (bosques, matorrales y humedales), agrícolas y forestales, deteriorando el estado y funcionamiento ambiental de estos territorios (Romero y Vásquez, 2005a).

La planificación urbana está relacionada con el rol del Estado a distintas escalas para intervenir con el diseño, mantenimiento y administración de las ciudades (Maantay, J., y Ziegler, J.,2006). El incremento de la población, el crecimiento urbano, el acelerado proceso de urbanización, y sus repercusiones sobre el medio natural, han provocado a lo largo de la historia desequilibrios ambientales que se convierten en temas recurrentes de diagnóstico y análisis (Cifuentes Ruiz, 2009). Los procesos de crecimiento urbano se presentan en el espacio-tiempo, ocasionando impactos agresivos al medio ambiente derivándose una gran cantidad de problemas ambientales, que, en ocasiones, resultan impredecibles (Alberto, J. 2009). Los cambios económicos, sociales y la urbanización de un 
territorio, en la mayoría de las ocasiones están ligados al desarrollo industrial (Rojas, Muñiz y Garcia, 2009). Estos factores han hecho que la ciudad se transforme en un espacio dinámico y en continuo crecimiento, donde la demanda de recursos es cada vez mayor.La urbanización como proceso, tanto en número de urbes como en cantidad de habitantes, es un fenómeno social demográfico con un fuerte impacto sobre el ambiente.

\section{OBJETIVOS}

Evaluar como la alteración del paisaje histórico cultural ha ocasionado la elevada sensibilidad a la intervención en su calidad visual, en el cerro Intiorko, distrito Alto de la Alianza de la ciudad Tacna del 2003-2017.
Evaluar como la ocupación informal del territorio se incrementa mediante el cambio del paisaje con relación al tiempo en el cerro Intiorko, distrito Alto de la Alianza de la ciudad Tacna del 2003-2017.

\section{METODOLOGÍA}

Investigación básica, no experimental correlacional, descriptivo explicativo donde se buscará medir la relación causa efecto entre las variables Proceso de ocupación informal del territorio y la Alteración del paisaje histórico cultural. El área de estudio se localiza en el Cerro Intiorko, correspondiente al distrito Alto de la Alianza, considerando los vestigios de la Cripta de los Héroes representando el paisaje Histórico Cultural, por involucrar las actividades protocolares en honor a los caídos en la Guerra del Pacifico de 1880, durante los años 1901 hasta
1979, ver apéndice B de la ciudad de Tacna, Perú.Las unidades de estudio están conformadas por los lotes de las asociaciones pecuarias así como las sociedades representativas de la sociedad de Tacna. La muestra está conformada por 288 parcelas de asociaciones pecuarias, según el plano catastral obtenido por la Municipalidad Distrital de Alto de la Alianza. Además de 60 encuestas realizadas a las sociedades representativas para la realización de la evaluación del paisaje en su calidad visual por el método directo.

\section{RESULTADOS}

A raíz de la evaluación de las vistas aéreas del área de estudio y el plano catastral de la Municipalidad Distrital de Alto de la Alianza, es

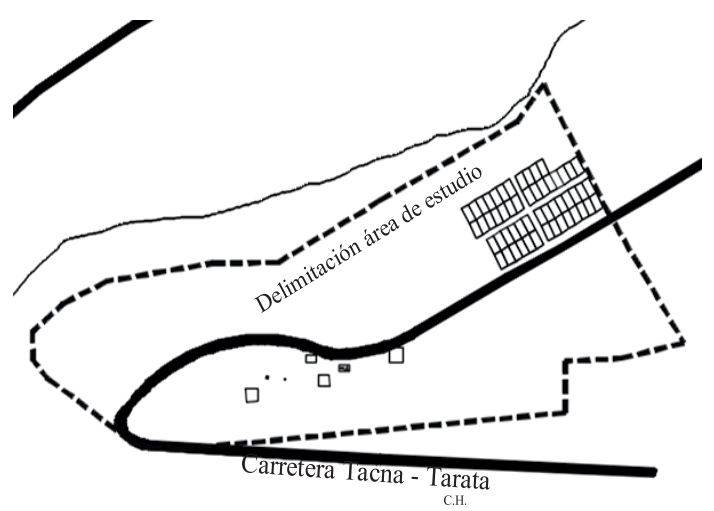

Fig 01: Área de ocupación año 2006 - 2008: Se aprecia el incremento de lotes correspondiente a la Asociacion de criadores de animales menores la Cruz Divina; frente a esta se ubica los lotes correspondientes a la Asoc. de pequeños criadores de aves Alto Santa Cruz. que se obtuvieron los siguientes resultados, para la ocupación del territorio y la evaluación de la vegetación.

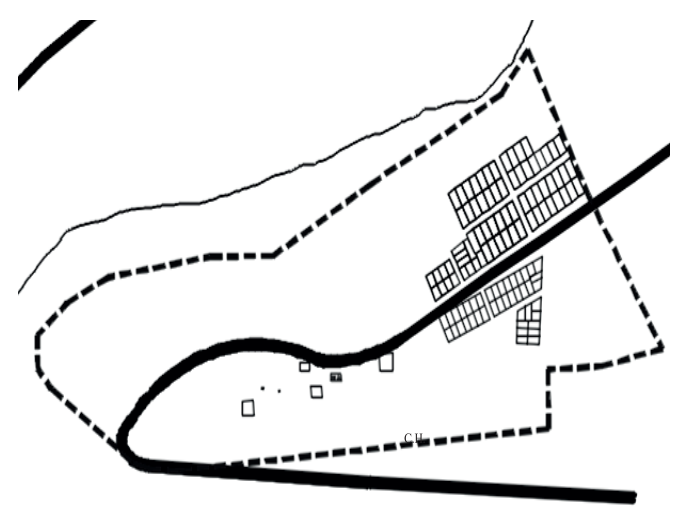

Fig. 02.Área de ocupación año 2009: Se aprecia el incremento de lotes correspondiente a las asociaciones de criadores de animales menores la Cruz Divina; y la Asoc. de pequeños criadores de aves Alto Santa Cruz. 


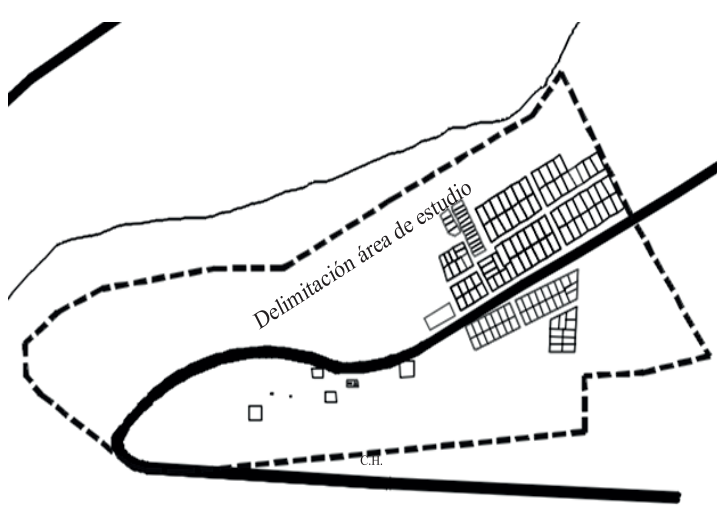

Fig. 03.Área de ocupación año 2010: se aprecia mayor consolidación en los lotes correspondientes a las asociaciones de criadores de animales menores la cruz divina; y la asoc. de pequeños criadores de aves alto santa cruz.

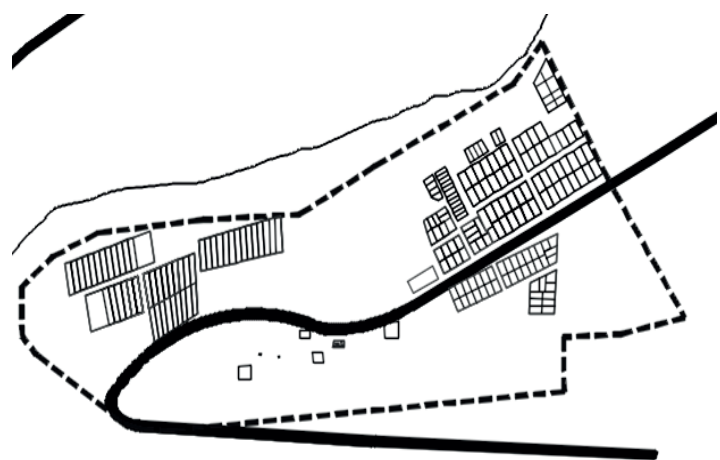

Fig.05. Área de ocupación año 2012: se aprecia los lotes correspondientes a las asociaciones de criadores de animales menores la cruz divina; y la asoc. de pequeños criadores de aves alto santa cruz y hacia el oeste mayor a la asociación de transportistas siempre unidos.

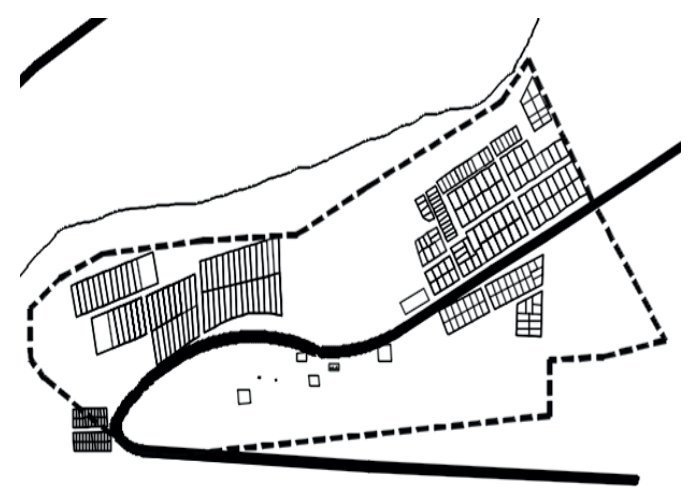

Fig. 07. Área de ocupacion 2014-2015: Se aprecia el incremento en lotes en las asociaciones de criadores de animales menores la Cruz Divina; Asoc. pequeños criadores de aves Alto Santa Cruz y la Asociación de transportistas Siempre Unidos junto con la mayor delimitación en vias locales de comunicación para su accesibilidad.

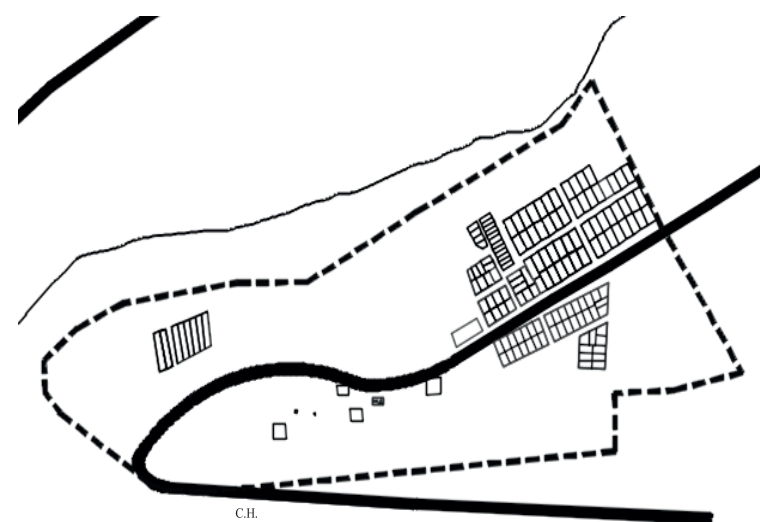

Fig. 04: área de ocupación año 2011 :se aprecia los lotes correspondientes a las asociaciones de criadores de animales menores la cruz divina; y la asoc. de pequeños criadores de aves alto santa cruz y hacia el oeste la delimitacion de lotes corresondientes a la asociación de transportistas siempre unidos.

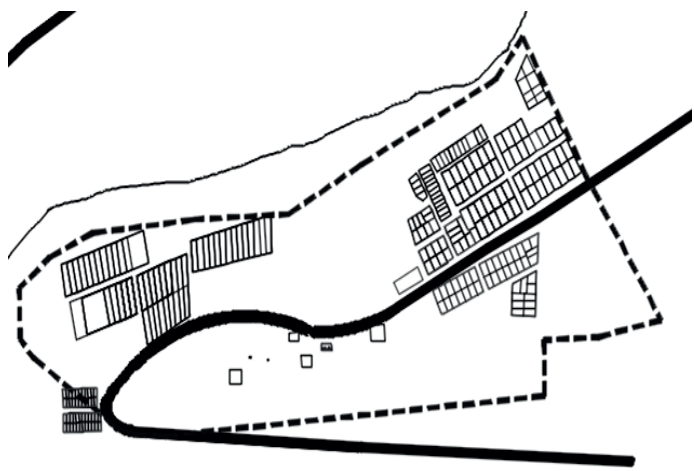

Fig. 06. Area de ocupación 2013: Se aprecia los lotes correspondientes a las asociaciones de criadores de animales menores la Cruz Divina; y la Asoc. de pequeños criadores de aves Alto Santa Cruz y hacia el oeste mayor consolidacion en la delimitacion de lotes corresondientes a la Asociación de transportistas Siempre Unidos

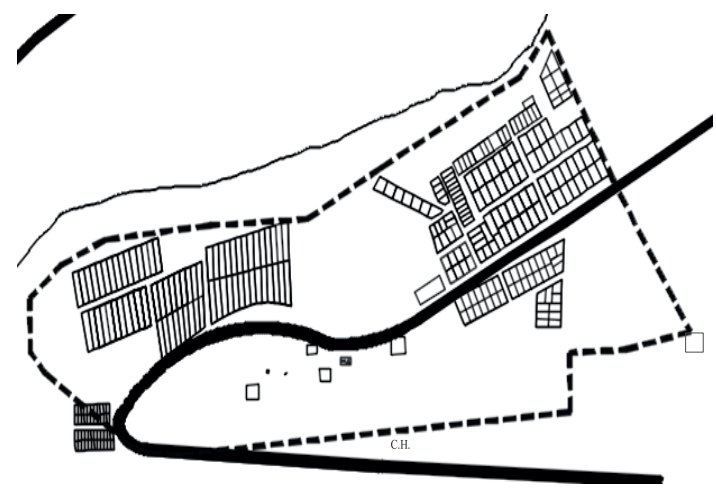

Fig. 08: Área de ocupación 2016: Se aprecia los lotes correspondientes a las asociaciones de criadores de animales menores la Cruz Divina; la Asoc. de pequeños criadores de aves Alto Santa Cruz y Asociación de transportistas Siempre Unidos; la delimitacion con cerco de lotes hacia el este de la Asoc. de Vivienda taller Cristo en el mirador. 


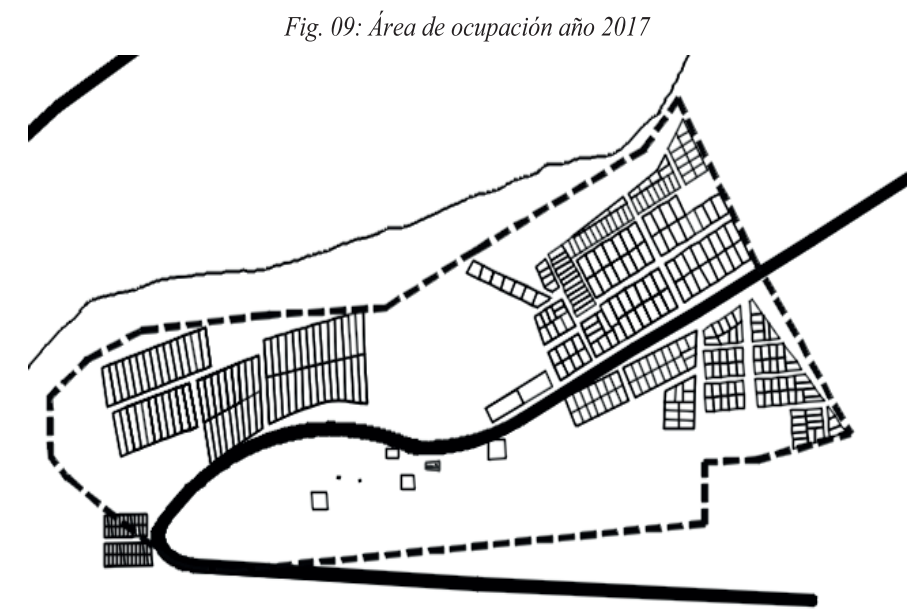

Asimismo, se puede apreciar en la figura 10 , se puede apreciar que el incremento de la ocupación del territorio, ocasiona la disminución de la ocupación de la vegetación en el área de estudio del Cerro Intiorko, esto producto de una ocupación sin una planificación territorial adecuada que responda al entorno ya que dentro del paisaje se encuentra ubicado la Cripta de los Héroes.

Fig. 10: Ocupación vegetación

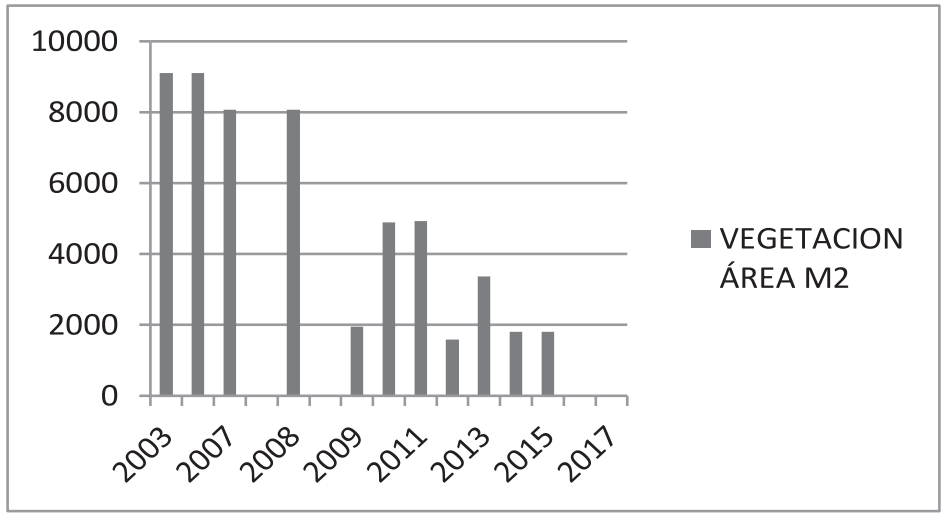

Método indirecto: Calidad visual de cada unidad

Método indirecto: Calidad visual de cada unidad

TABLA 01: Evaluación de las unidades de paisaje según el método indirecto

\begin{tabular}{|c|c|c|c|c|c|c|c|}
\hline \multirow{2}{*}{$\begin{array}{l}\text { Unidad } \\
\text { de } \\
\text { paisaje }\end{array}$} & \multicolumn{5}{|c|}{ Componentes visuales del fondo escénico } & \multirow[t]{2}{*}{ sumatoria } & \multirow{2}{*}{$\begin{array}{l}\text { Normali } \\
\text { zado } 1-5\end{array}$} \\
\hline & morfología & vegetación & color & $\begin{array}{l}\text { singularida } \\
\text { d }\end{array}$ & $\begin{array}{l}\text { Actuaciones } \\
\text { humanas }\end{array}$ & & \\
\hline $\mathrm{A}$ & 5 & 3 & 3 & 6 & 0 & 17 & 3.74 \\
\hline B & 3 & 3 & 1 & 2 & 0 & 9 & 2.05 \\
\hline
\end{tabular}

Tabla 02: Resultados Método Indirecto

\begin{tabular}{ccc}
\hline Unidad de paisaje & Ponderado & Calidad visual \\
\hline $\mathrm{A}$ & 3.74 & Media - alta \\
\hline $\mathrm{B}$ & 2.05 & Media - baja \\
\hline
\end{tabular}

Los resultados obtenidos denotan que la unidad Aes más relevante, cuenta con la ubicación de la Cripta de los Héroes, su calidad visual es media - alta; elemento de gran singularidad dentro del conjunto del territorio en estudio, dadas sus características geomorfológicas y su singularidad. La unidad B, presenta una menor relevancia por su calidad visual: media - baja, ya que sus rasgos no son tan singulares, la vegetación se vio afectada por las asociaciones (tabla 01 y 02). 


\section{Método Directo: calidad visual de cada unidad.}

Tabla 03: Resultados método directo

\begin{tabular}{llll}
\hline Unidad de paisaje & imagen & ponderado & Calidad visual \\
\hline $\mathrm{A}$ & Imagen 1 & 3.78 & Media - alta \\
\hline $\mathrm{A}$ & Imagen 2 & 2.91 & media \\
\hline $\mathrm{A}$ & Imagen 3 & 0.56 & baja \\
\hline $\mathrm{B}$ & Imagen 4 & 0.64 & baja \\
\hline $\mathrm{B}$ & Imagen 5 & 0.72 & baja \\
\hline
\end{tabular}

Fig. 11: Gráfico de secuencia del número de lotes

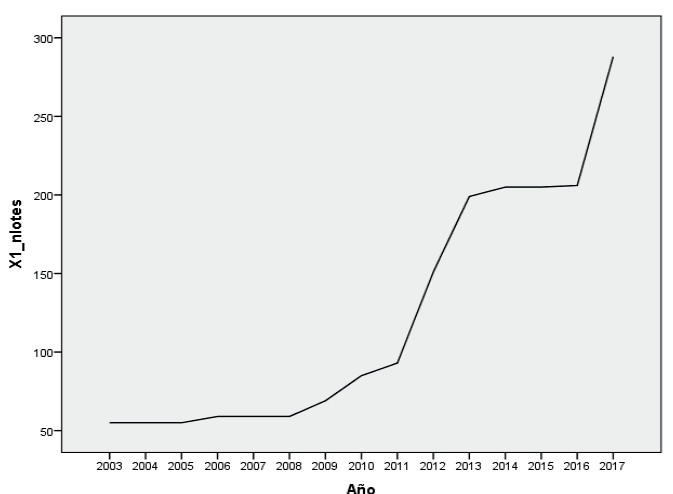

La figura 11 permite apreciar la evolución del número de lotes a través del periodo de análisis, observándose un crecimiento con mayor énfasis a partir del año 2011, afectando al área vegetal de la zona de estudio. Este incremento vertiginoso del número de lotes se debe al incremento desmesurado de la población en Tacna, debido a los fenómenos demográficos del proceso de ocupación del territorio como es la migración.

Fig. 13: gráfico de secuencia de área vegetal.

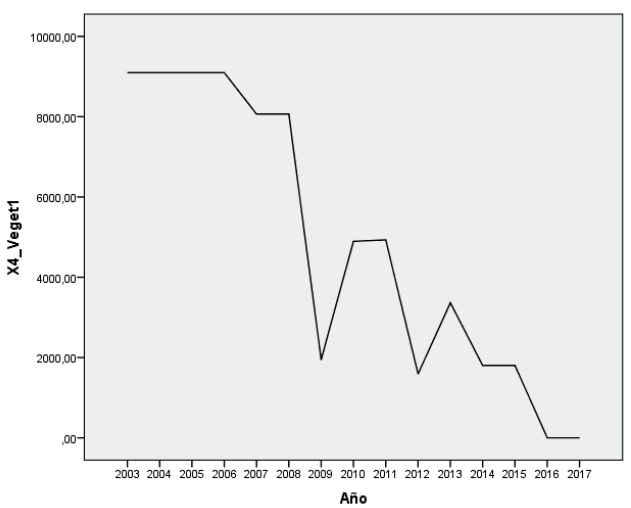

La figura 13 permite apreciar la evolución del desarrollo del área vegetal a través del periodo de análisis, apreciándose la reducción del área de ocupación debido a la influencia del área de ocupación antrópica, presentando un ligero incremento entre el 2010 y 2013.
FIG. 12: Gráfico de Secuencia de Área Ocupada.

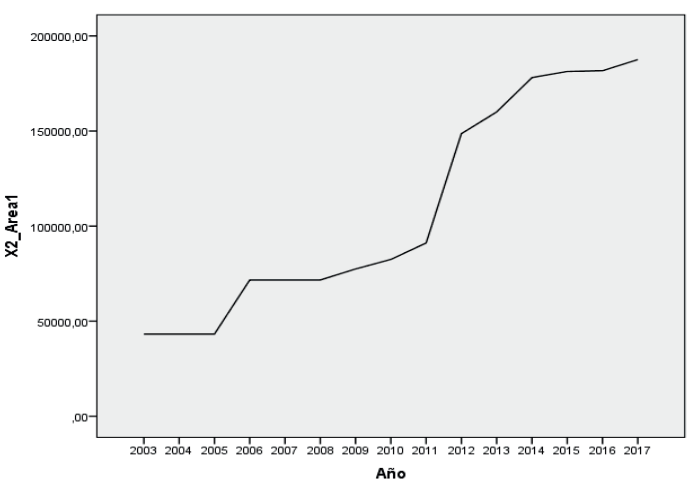

La figura 12 permite apreciar la evolución del área ocupada a través del periodo de análisis, observándose un crecimiento, el mismo que afecta al área vegetal de la zona de estudio.

Fig. 14: Secuencia de calidad visual paisaje natural

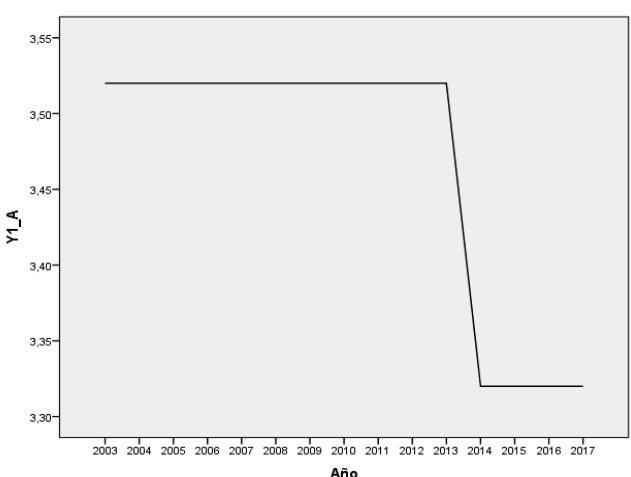

La figura 14, permite apreciar el desarrollo de la calidad visual del paisaje natural, resultado de la evaluación a través de categorías estéticas por el método indirecto obteniendo una calidad visual MEDIA - ALTA, que denota que el componente de la unidad natural es relevante de gran singularidad dentro del conjunto del territorio en estudio. 


\section{DISCUSIÓN}

Se ha determinado la influencia del proceso de ocupación informal del territorio en el paisaje histórico cultural en el cerro Intiorko en función que el incremento en la ocupación del territorio disminuye la calidad visual del paisaje, originando su alteración; obteniendo resultados en la calidad visual del paisaje para el área natural de MEDIA -ALTA y para el área ocupada de BAJA; mediante su evaluación por el método indirecto, considerando el incremento de la ocupación del territorio y la disminución del área de vegetación de la flora endémica. La evaluación del paisaje histórico cultural en el cerro Intiorko, se realizó a través de la técnica aplicada por el Bureau of Land Management (BLM) de estados Unidos, método de evaluación indirecto, la misma que consiste en valorar cuantitativamente las unidades homogéneas de diversos aspectos, obteniendo una calidad visual MEDIA - ALTA, correspondiente al área natural, debido a que cuenta con el aspecto de singularidad, al contener la Cripta de los Héroes, permitiendo considerar el objetivo de calidad del paisaje de clase $A$, de conservación y mantenimiento y una calidad visual BAJA para el área de ocupación, considerando así el objetivo de calidad del paisaje sea de clase $\mathrm{E}$, de máxima modificación del carácter existente.

La ocupación informal del territorio estudiado tuvo un incremento a partir del año 2010, con el incremento de lotes destinados a la actividad pecuaria, el mismo que influye en la disminución del área de ocupación de la vegetación, flora endémica y de la BAJA calidad visual del paisaje.
Cabe mencionar que el territorio de estudio en el plan director 2010, era un área de protección ecológica, para el PAT 2015 - 2025 esta se encuentra definida como área para la actividad pecuaria, el mismo que presenta riesgo alto para la actividad residencial.

Se hace necesario valorar el Paisaje Histórico Cultural, del cerro Intiorko, considerando patrimonio inmueble histórico a la Cripta de los Héroes, por parte del Ministerio de Cultura, ya que por los resultados obtenidos se requiere su conservación total, lo que permitirá convertir el lugar en un destino turístico diferenciado de otros que revalore el paisaje histórico, capaz de atraer mayor número de turistas y visitantes.

Por ello se debe contar con el apoyo de la Dirección de Paisaje Cultura, a fin de delimitar el espacio de la Cripta de los Héroes como parte del paisaje histórico cultural, legajo del enfrentamiento de 1880, como área intangible. La ocupación del territorio debe ceñirse a no permitir que dicha ocupación se realice en zonas de alto riesgo, alta vulnerabilidad y de protección ecológica, contando con un instrumento de planificación territorial que sea sostenible, todo ello debe ser verificado por la Municipalidad Provincial de Tacna, así como el Gobierno Regional de Tacna. Mejorar los instrumentos para la protección ecológica, a fin de que sean respetados y no modificados sin argumentos técnicos sostenibles, ya que la flora del lugar es endémica, Tillandsia Werdermannii, "Siempre Viva" es patrimonio regional.

\section{REFERENCIAS BIBLIOGRÁFICAS}

Alberto, J. A. (2009). Geografía y Crecimiento Urbano. Paisajes y Problemas Ambientales. G e o g r á fi c a D i g i t a I, https://www.sistemamid.com/panel/uploads/bi blioteca/7097/7098/7110/7111/82675.pd

Aponte, Gloria (2003). Paisaje e identidad cultural. Tabula Rasa. Bogotá - Colombia, N.1: 153164, enero- diciembre de 2003.

Basadre, Jorge. Historia de la República del Perú. (1822-1933).

Cifuentes Ruiz, Biodiversidad de Tenebrionidae (Insecta: Coleoptera) en México, 2009. URL: http://www.elsevier.es/es-revista-revistamexicana-biodiversidad-91-articulobiodiversidad-tenebrionidae-insectacoleoptera-mexico-S1870345314707080

Diccionario de la real lengua española, 1992. URL:http://www.rae.es/recursos/diccionarios/ diccionarios-anteriores-17262001/diccionario-de-la-lengua-espanola2001.

García E. (2008). El proceso de expansión urbana y su impacto en el uso de suelo y vegetación del Municipio de Juárez, chihuahua (tesis de maestría). Colegio de la Frontera Norte, México

Maantay, J., y Ziegler, J. (2006). GIS for the Urban Environment. Redlands California: ESRI Press.

Masmela, Paula. el paisaje como elemento de la ordenación territorial. un análisis de paisaje

Morales A. (1991) El Calvario de Tacna. Lima: J. Morales Arias. desde su enfoque visual en el borde centro oriental de Medellín, Colombia $2010 \quad$ U R L : http://www.bdigital.unal.edu.co/3734/1/32143 
590.2011 1.pdf

Moschella P. (2012) Variación y protección de humedales costeros frente a procesos de urbanización: casos Ventanilla y Puerto Viejo (tesis maestría). Pontificia Universidad Católica del Perú

Pacheco, C. (2015) La experiencia estética en y desde el paisaje natural chileno (tesis de maestría). PONTIFICIA UNIVERSIDAD CATÓLICA DE CHILE. Recuperada de la base de datos del Sistema de biblioteca de la Pontificia Universidad católica de Chile.

Pérez, L; Parra, C; (2004). PAISAJES CULTURALES:

EL PARQUE PATRIMONIAL COMO INSTRUMENTO DE REVALORIZACIÓN Y REVITALIZACIÓN DEL TERRITORIO. Theoria,13 9-24. Recuperado de http://www.redalyc.org/articulo.oa?id=299013 02

Rojas, Muñiz y García, 2009. Estructura urbana y policentrismo en el Área Metropolitana de Concepción. Recuperado de http://www.scielo.cl/scielo.php?script=sci_artt ext\&pid=S0250-71612009000200003

Romero, H., Molina, M., Moscoso, C., Sarricolea, P., Smith, P. Y Vasquez, A. (2007). Caracterización de los cambios de usos y coberturas de suelos causados por la expansión urbana de Santiago, análisis estadístico de sus factores explicativos e inferencias ambientales. En DE MATTOS C., HIDALGO R. (Editores), Santiago de Chile, Movilidad Espacial y Reconfiguración Metropolitana. pp. 251-270.

Sánchez, A.A. (2015) Migraciones internas en el Perú. $R$ e c u p e r a d o d e http://www.oimperu.org/sitehome/sites/default / fi I es/Documentos/03-032015_Publicacion\%20Migraciones\%20Intern as OIM.PDF

Villarino $\bar{M}$. (1985). Curso sobre evaluación de impacto ambiental. Dirección general del Medio Ambiente M.O.P.U. Madrid

Presentado: Marzo 2017

Aceptado: Junio 2017 